MATEC Web of Conferences 22,03017 (2015)

DOI: $10.1051 /$ matec conf/ 20152203017

(C) Owned by the authors, published by EDP Sciences, 2015

\title{
Design and Realization of Fitness Simulation Horse Based on Control Theory
}

\author{
Lei Geng* \& Gang Wu \\ Department of Public Fundation, Langfang Health Vocational College, Langfang, Hebei, China
}

\begin{abstract}
As people's living standard improves, they are increasingly concerned with health, and researches on various kinds of sports apparatus are also growing, such as the fitness simulation horse. In this paper, we first described the advantages of the fitness simulation horse to the human body; then designed a fitness simulation horse platform with a new orthogonal parallel mechanism; set up the control system of fitness simulation horse in an open structure; established a control model of fitness simulation horse, a structure model for branched-chain control and a controller model based on the kinematics; validated these models in simulation experiments; and last made a prospect on future development.
\end{abstract}

Keywords: Control theory; Fitness simulation horse; Kinematics model

\section{INTRODUCTION}

The bionics has been highly focused at home and abroad in recent years, and related research institutions have been founded in many universities and scientific research institutes. The US and Japan have kept ahead in their researches on bionics over the world, whereas China is relatively backward. The bionics is generated from the inspiration brought by the creatures in nature. Increasingly growing bionics researches have been made in the engineering field, and certain achievements have been obtained.

Wang $\mathrm{Ou}$, when studying the horse, was inspired to construct a bionic horse. He used the MCU and a controller to build a bionic horse robot, tested it through simulation, and then proved the feasibility of its electromechanical control system. Zhang Shouquan applied the bionics into the mechanical design, and discussed the functions and structures and broke a new ground of bionics researches in the engineering field. Zhang Wei et al. analyzed the kinematics of a running horse, defined its kinematic sketch based on bionics, and then analyzed all contact points on the horse body to successfully prove the feasibility and rationality of the designed system for a running horse. Zhao Jingbo analyzed the bionic robot horse, set up a 6DOF kinematics model, and finally validated the correctness and rationality of this model through simulation. Ren Yuyan, when studying the relationship between the physical changes and physiological changes in the human body during movement, analyzed the effects of the bionic horse on the body functions during movement, improved the ability of the bionic horse to receive a variety of information at the learning stage by changing the algorithm, and finally realized the real-time control on the fitness simulation horse during the experiment.

This paper, based on the results of previous re-

*Corresponding author: 35372989@,qq.com searches, constructed a fitness simulation horse based on the control theory, set up a control platform of fitness simulation horse by analyzing the kinematics principles of human body and horse and then validated this control platform in the experiment, thus laying the theoretical foundation for the implementing this control platform.

\section{CONTROL DESIGN AND IMPLEMENTATION OF FITNESS SIMULATION HORSE}

As limited by various conditions, few people can ride a horse in real life, but more people prefer to ride a simulation robot horse to keep fit. Although the researches on this robot are now growing, but most of them are still at the beginning stage. For this reason, the fitness simulation horse was further studied in this paper based on these previous researches.

\subsection{Mechanism Design of Fitness Simulation Horse}

A good structural performance is needed to obtain the rationality and stability of actions made by the fitness simulation horse. As a result, this paper set up an improved Steward Platform mechanism model to reduce the mechanism complexity.

The improved Steward platform is a 3D-platform parallel mechanism, mainly consisting of the base, branched chains and platform, as shown in the figure 1.

Main characteristics of this platform layout include:

1) Three branched chains are respectively perpendicular to the motion platform, and the applied parallel mechanism has higher bearing capacity and is characterized by good distortionless coupling.

2) The hinge points on the motion platform are also the connecting points on the base, similar to the translational pair. 


\section{MATEC Web of Conferences}

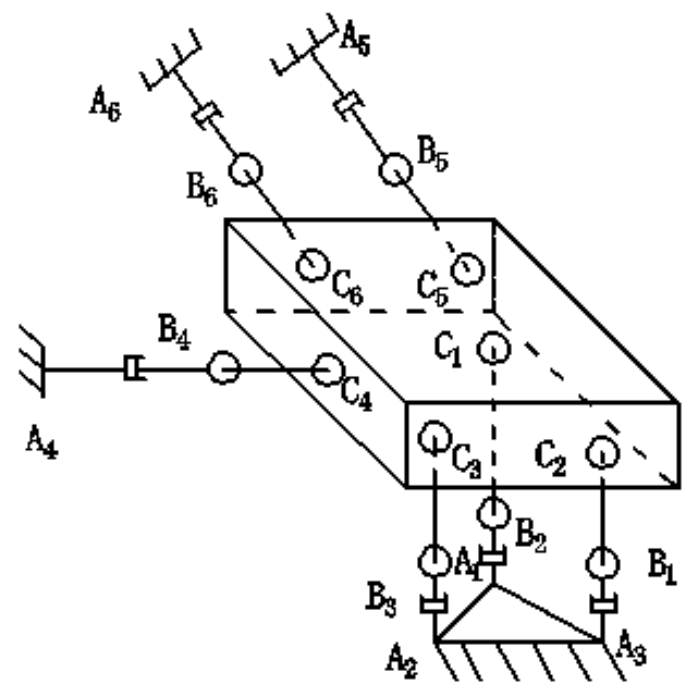

Figure 1. Fitness Horse Structure Simulation Platform

3) Every branched chain constitutes a branch composed of the translational pair, connecting rod and ball joint. The hinge center on the motion platform is perpendicular to the platform, and the ball joint of every branched chain is centered on the corresponding platform.

\subsection{Control System of Fitness Simulation Horse}

In order to achieve a stable system, quick response and high precision control, the structure of fitness simulation horse needs to be analyzed and studied. As it is a multi-axis real-time motion control system, the effectively way to assure the coordination among all kinematic branched chains for associated movement is to analyze the system functions and subsequently set up reasonable and valid control platform.

\subsubsection{Control Theory Principles of Fitness Simula- tion Horse}

The fitness simulation horse is designed through inte- gration and development of current techniques rather than implemented by the designer itself by every piece. Therefore, proper plates and controllers may be selected to be integrated and a control system is developed in the design of fitness simulation horse, so as to achieve high precision, quick response and good stability. In view of this, the control theory of fitness simulation horse needs to meet following requirements:

1) Quite able to control the points, and also improve the expansion and openness capabilities and enhance the flexibility and freedom as actually required;

2) 6 branched chains of the fitness simulation horse are mutually coordinated, completely controlled by the control system, and strongly sensitive and quickly responsive to motion commands;

3) The human-machine interface is fitted properly. As the connection between the fitness simulation horse and the human body, it not only can simulate the state of motion of fitness simulation horse to grasp its current movement condition, but also can be operated to control the system.

\subsubsection{Control System Structure of Fitness Simulation} Horse

Based on the control theory above, the multi-axis motion controller and the $\mathrm{NC}$-embedded PC structure are applied to building up the motion control system, which can reduce the cost and improve the integration between open mode structure and information.

Three systems are constructed for the fitness simulation horse by the functional needs and structures drive system, control system and user system, among which the structure of control system is shown in the figure 2 .

1) Drive system: Realize the motion of fitness simulation horse as ordered by the command from control system; meanwhile, the detection information can be fed back to the inside of drive system to control the motor position and speed.

2) Motion control system: This system is mainly to generate the trajectory of fitness simulation horse and process the host commands, realizing the host movement with information feedback and thus processing a variety of information.

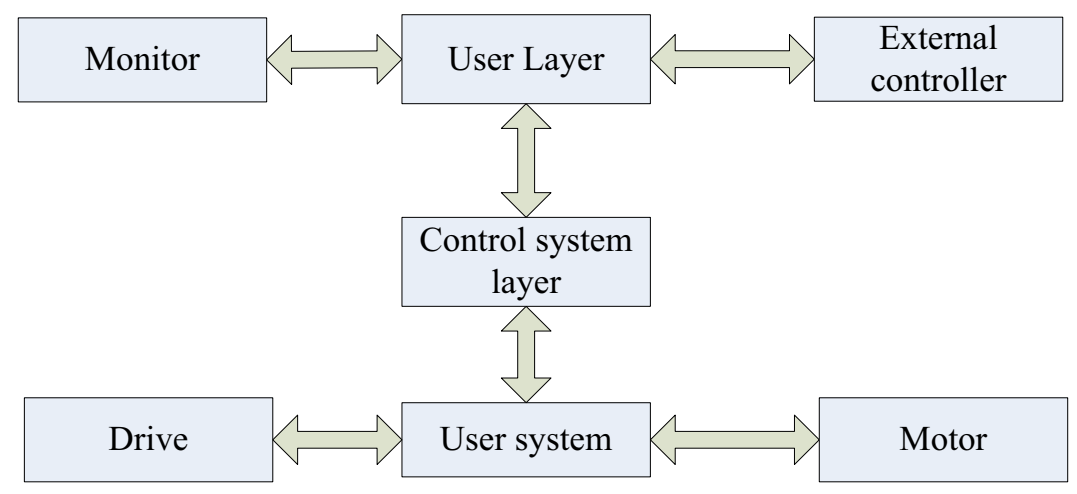

Figure 2. Fitness Horse Simulation Control Structure of Hardware Platform 
3) User system: This system bridges the information between a host and fitness simulation horse and then exchanges the information to run the system. In addition, it can also calculate the motion position and state of the fitness simulation horse to execute the user's control commands.

\section{MOTION CONTROL MODEL OF FITNESS SIMULATION HORSE}

The kinematics theory is the important foundation for constructing a fitness simulation horse, mainly concerning the relationship between the joint end trajectory and joint variables. With all joint parameters assumed to be known, the end posture of fitness simulation horse can be identified based on kinematics, and when the end effector is determined and specially postured, the parameters of every joint can also be identified based on kinematics, so that the movement of fitness simulation horse is precisely controlled. This shows the establishment of kinematical equations for the fitness simulation horse is of great significance to the control theory study.

\subsection{Rigid Body Transformation Model}

Generally, the used 3D coordinate system of rigid body is Cartesian coordinate system. Suppose the position of any one point in the space is $Q$, the corresponding vector is ${ }^{A} Q$, the rectangular coordinate system is $\{\mathrm{A}\}$ and positions of point ${ }^{A} Q$ respectively corresponding to axes $\mathrm{X}, \mathrm{Y}$ and $\mathrm{Z}$ are $\mathrm{Q}_{\mathrm{X}}, Q_{\mathrm{Y}}, Q_{\mathrm{Z}}$, and then the position vector ${ }^{A} Q$ can be represented as:

$$
{ }^{A} Q=\mathrm{Q}_{\mathrm{X}} i+Q_{\mathrm{Y}} j+Q_{\mathrm{Z}} k
$$

${ }^{A} Q$ represented in the matrix form is as follows:

$$
{ }^{A} Q=\left[\begin{array}{c}
\mathrm{Q}_{\mathrm{X}} \\
Q_{\mathrm{Y}} \\
Q_{\mathrm{Z}}
\end{array}\right]=\left[\begin{array}{lll}
\mathrm{Q}_{\mathrm{X}} & Q_{\mathrm{Y}} & Q_{\mathrm{Z}}
\end{array}\right]^{T}
$$

In order to identify the position of $B$ in the space, another coordinate system $\{B\}$ needs to be established, and then the corresponding unit vectors are $X_{B}, Y_{B}, Z_{B}$. Link these two coordinate systems, and then the corresponding equation is:

$$
{ }_{B}^{A} R=\left[\begin{array}{lll}
{ }^{A} X_{B} & { }^{A} Y_{B} & { }^{A} Z_{B}
\end{array}\right]
$$

Where, ${ }_{B}^{A} R$ is a $3 \times 3$ rotation matrix including three independent elements, with corresponding constraints given as follows:

$$
\begin{aligned}
& { }^{A} X_{B} \cdot{ }^{A} X_{B}={ }^{A} Y_{B} \cdot{ }^{A} Y_{B}={ }^{A} Z_{B} \cdot{ }^{A} Z_{B}=1 \\
& { }^{A} X_{B} \cdot{ }^{A} Y_{B}={ }^{A} Y_{B} \cdot{ }^{A} Y_{B}={ }^{A} Z_{B} \cdot{ }^{A} X_{B}=0
\end{aligned}
$$

Therefore, ${ }_{B}^{A} R$ is an orthogonal matrix, and its product of inverse matrix and transpose is 1 , that is:

$$
{ }_{B}^{A} R^{-1}={ }_{B}^{A} R^{T} \quad\left|{ }_{B}^{A} R^{T}\right|=1
$$

The rotation transformation of any kinds is obtained from the basic rotation transformation of axes $\mathrm{X}, \mathrm{Y}, \mathrm{Z}$, and the basic rotation transformation matrices of rotation angle $\theta$ are:

$$
\begin{aligned}
& R(X, \theta)=\left[\begin{array}{ccc}
1 & 0 & 0 \\
0 & \cos \theta & -\sin \theta \\
0 & \sin \theta & \cos \theta
\end{array}\right] \\
& R(\mathrm{Y}, \theta)=\left[\begin{array}{ccc}
\cos \theta & 0 & \sin \theta \\
0 & 1 & 0 \\
-\sin \theta & 0 & \cos \theta
\end{array}\right] \\
& R(\mathrm{Z}, \theta)=\left[\begin{array}{ccc}
\cos \theta & -\sin \theta & 0 \\
\sin \theta & \cos \theta & 0 \\
0 & 0 & 1
\end{array}\right]
\end{aligned}
$$

In the above equations, the position vector is used to identify the point position, and the rotation matrix is also used to identify the orientation of rigid body. The spatial position and posture of the object is determined by linking the coordinate system and object together, and the origin of the coordinates is generally selected on the object characteristic point. Take $\{\mathrm{B}\}$ as the example, and then the rigid body $B$ is in $\{\mathrm{B}\}$ :

$$
\{\mathrm{B}\}=\left\{{ }_{B}^{A} R \quad{ }^{A} Q_{B 0}\right\}
$$

When ${ }^{A} Q_{B 0}=0$, it refers to the object direction; when ${ }_{B}^{A} R=I$, it refers to the object position.

The vectors of the spatial point $p$ coordinate system are ${ }^{A} Q$ and ${ }^{B} Q$. The corresponding transformation relationship between them is:

$$
{ }^{A} Q={ }_{B}^{A} R^{B} Q+{ }^{A} Q_{B 0}
$$

Although the equation above is a non-homogeneous equation, it can be represented as a homogeneous one given below:

$\left[\begin{array}{c}{ }^{A} Q \\ 1\end{array}\right]=\left[\begin{array}{cc}{ }_{B}^{A} R & { }^{A} Q_{B 0} \\ 0 & 1\end{array}\right]\left[\begin{array}{l}{ }^{B} Q \\ 1\end{array}\right]$

The equation above shows a $4 \times 13 \mathrm{D}$ coordinate points in the homogeneous form, which can be denot- 


\section{MATEC Web of Conferences}

ed as ${ }^{A} Q$ or ${ }^{B} Q$, and then the corresponding matrix is:

$$
{ }^{A} Q={ }_{B}^{A} T^{B} Q
$$

The homogeneous matrix ${ }_{B}^{A} T$ is then transformed into a $4 \times 4$ matrix, as shown below:

$$
{ }_{B}^{A} T=\left[\begin{array}{cc}
{ }_{B}^{A} R & { }^{A} Q_{B 0} \\
0 & 1
\end{array}\right]
$$

\subsection{Inverse Kinematics Model}

RPY angle coordinate is 3D-transformed to represent the motion platform, and the corresponding position parameter is $q=\left(\begin{array}{llllll}x & y & z & \alpha & \beta & \gamma\end{array}\right)$. Where, Euler transformation angle of motion platform is $\alpha, \beta, \gamma$, and the coordinate in the coordinate system $\{\mathrm{W}\}$ is $\mathrm{x}, y, z$. To simplify the calculation process, the coordinate is transformed as:

$$
R=R_{x}+R_{y}+R_{z}
$$

From the equation above, the ball joint in the coordinate system $\{\mathrm{W}\}$ can be represented as:

$$
C_{i}^{\prime}=R C_{i}+(\mathrm{x}, \mathrm{y}, \mathrm{z})^{T}
$$

Where, $B_{i}, C_{i}$ are known, and then the constraint can be accordingly obtained from the geometrical relationship:

$$
\left|B_{i} C^{\prime}\right|=L
$$

From the two-point distance formula, the functional equations of time $t$ for $\mathrm{x}, y, z, \alpha, \beta, \gamma$ and $l$ can be obtained, corresponding to each branched chain as follows:

Branched Chain 1:

$\left(\mathrm{x}-\mathrm{ap}_{x}-\mathrm{gm}_{x}+\mathrm{a}\right)^{2}+\left(\mathrm{y}-\mathrm{ap}_{y}-\mathrm{gm}_{y}+\mathrm{H}_{2}-l_{6}\right)^{2}$

$+\left(\mathrm{z}-\mathrm{ap}_{z}-\mathrm{gm}_{z}-\mathrm{h}-\mathrm{c}\right)^{2}=L^{2}$

Branched Chain 2:

$\left(\mathrm{x}+\mathrm{ap}_{x}-\mathrm{gm}_{x}-\mathrm{a}\right)^{2}+\left(\mathrm{y}+\mathrm{ap}_{y}-\mathrm{gm}_{y}+\mathrm{H}_{2}-l_{5}\right)^{2}$

$+\left(\mathrm{z}+\mathrm{ap}_{z}-\mathrm{gm}_{z}-\mathrm{h}-\mathrm{c}\right)^{2}=L^{2}$

Branched Chain 3:

$\left(\mathrm{x}-f m_{x}-H_{1}-l_{4}\right)^{2}+\left(\mathrm{y}-f \mathrm{p}_{y}\right)^{2}$

$+\left(z-f p_{z}-\mathrm{h}-\mathrm{c}\right)^{2}=L^{2}$

Branched Chain 4:

$\left(\mathrm{x}-b p_{x}+e m_{x}-c n_{x}-b\right)^{2}+\left(\mathrm{y}-b \mathrm{p}_{y}+e \mathrm{~m}_{y}+c n-e\right)^{2}$

$+\left(\mathrm{z}-b \mathrm{p}_{z}+e \mathrm{~m}_{z}-c n_{z}-l_{3}\right)^{2}=L^{2}$

Branched Chain 5: $\left(\mathrm{x}+b p_{x}+e m_{x}-c n_{x}-b\right)^{2}+\left(\mathrm{y}+b \mathrm{p}_{y}+e \mathrm{~m}_{y}-c n-e\right)^{2}$

$+\left(\mathrm{z}+b \mathrm{p}_{z}+e \mathrm{~m}_{z}-c n_{z}-l_{3}\right)^{2}=L^{2}$

Branched Chain 6:

$\left(\mathrm{x}-d m_{x}-c n_{x}\right)^{2}+\left(\mathrm{y}+d-d \mathrm{~m}_{y}-c n_{y}\right)^{2}$

$+\left(\mathrm{z}-d \mathrm{~m}_{z}-c n_{z}-l_{1}\right)^{2}=L^{2}$

Considering the initial posture, the motion output function of motion platform is turned to:

$R=E \quad x=0 \quad y=0 \quad z=h+c \quad l_{i}=q_{0}$

From the equations above, the expressions of motion inverse solutions for fitness simulation horse are:

$$
\begin{aligned}
& l_{1}=z-d m_{z}-c n_{z}+\left[\mathrm{L}^{2}-\left(\mathrm{x}+d \mathrm{~m}_{x}-\mathrm{cn}_{x}\right)^{2}\right. \\
& \left.-\left(\mathrm{y}-d m_{y}-\mathrm{cn}_{y}\right)^{2}-\left(\mathrm{y}-\mathrm{dm}_{y}-\mathrm{cn}_{y}+\mathrm{d}\right)^{2}\right]^{1 / 2} \\
& l_{2}=z+b p_{z}+e m_{z}-c n_{z}+\left[\mathrm{L}^{2}-\left(\mathrm{bp}_{x}+\mathrm{em}_{x}-\mathrm{cn}_{x}\right.\right. \\
& \left.+\mathrm{x}-\mathrm{b})^{2}-\left(\mathrm{bp}_{y}+\mathrm{em}_{y}-\mathrm{cn}_{y}+\mathrm{y}-\mathrm{e}\right)^{2}\right]^{1 / 2} \\
& l_{3}=z-b p_{z}+e m_{z}-c n_{z}+\left[\mathrm{L}^{2}-\left(\mathrm{x}+\mathrm{b}-\mathrm{bp}_{x}\right.\right. \\
& \left.\left.+\mathrm{em}_{x}-\mathrm{cn}_{x}\right)^{2}-\left(\mathrm{y}-\mathrm{bp}_{y}+\mathrm{em}_{y}-\mathrm{cn}_{y}-\mathrm{e}\right)^{2}\right]^{1 / 2}
\end{aligned}
$$

$l_{4}=x+H_{1}-f p_{x}+\left[\mathrm{L}^{2}-\left(y-f p_{y}\right)^{2}\right.$

$\left.-\left(\mathrm{z}-f p_{z}-h-\mathrm{c}\right)^{2}\right]^{1 / 2}$

$l_{5}=y+a p_{y}-g m_{y}+H_{2}-\left[\mathrm{L}^{2}-\left(\mathrm{x}+a \mathrm{p}_{x}\right.\right.$

$\left.\left.-g \mathrm{~m}_{x}-a\right)^{2}-\left(\mathrm{z}+a \mathrm{p}_{z}+g \mathrm{~m}_{z}-h-c\right)^{2}\right]^{1 / 2}$

$l_{6}=y-a p_{y}-g m_{y}+H_{2}-\left[\mathrm{L}^{2}-\left(\mathrm{x}+a \mathrm{p}_{z}+g \mathrm{~m}_{z}\right.\right.$

$\left.-z+h+c)^{2}-\left(a \mathrm{p}_{x}+g \mathrm{~m}_{x}-x-a\right)^{2}\right]^{1 / 2}$

This equation expresses the kinematical inverse solution of fitness simulation horse, through which it can be seen that the expression structure is unique and simple. Therefore, the design of orthogonal parallel mechanical to control the fitness simulation horse is reasonable and effective.

\subsection{Study on the Control of Motion Platform Branched Chains}

In order to easily install the fitness simulation horse, the semi-closed loop control was used in this paper to control the spatial position and assure sufficient precision, rather than the fully-closed loop control directly connecting the machinery to sensors.

As the running fitness simulation horse is on varied load, and its speed also varies and is not stable, the key is to control the current to make it run steadily. Considering the application of the current closed loop developed by Panasonic in the servo drive, a solution with control principle shown below was proposed in this paper: 


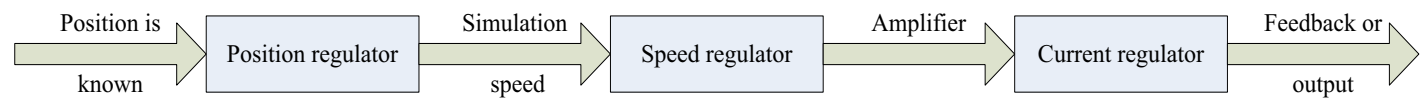

Figure 3. Motion Platform Branched Control Chart
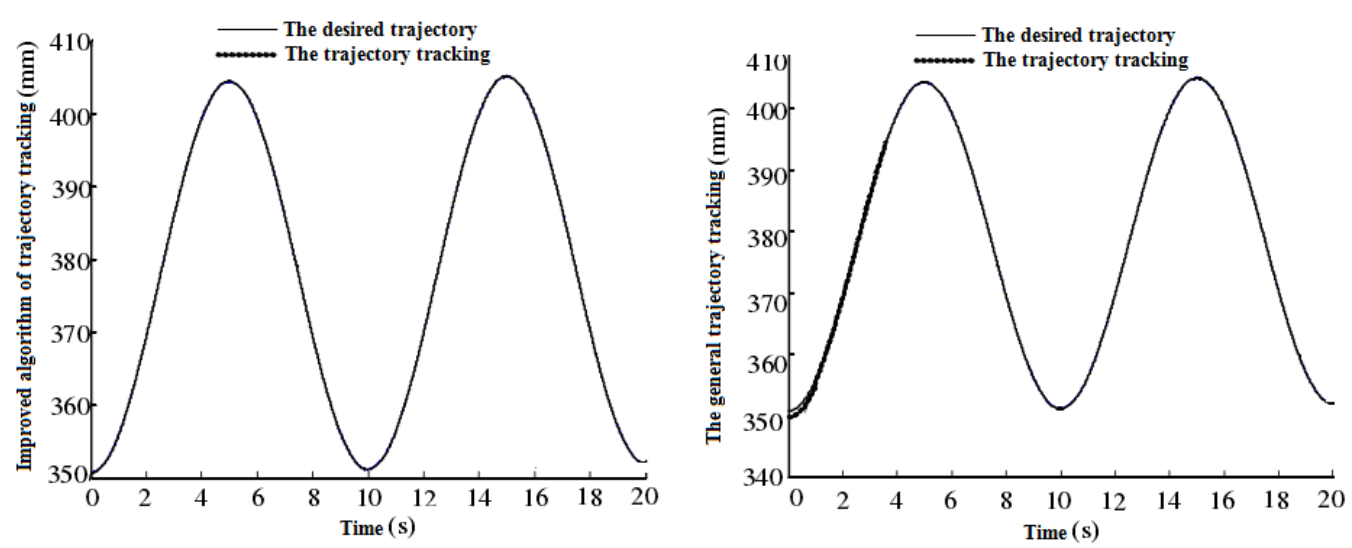

Figure 4. Comparison between Traditional Controller and Digital Simulation Controller

In this paper, in order to effectively control the position of fitness simulation horse, a three-layered closed loop circuit was used, arranged from outside to inside with position loop, speed loop and current loop, so as to lessen the current changes in the phase position and amplitude and also to effectively control the steadiness of the fitness simulation horse during the entire movement.

\section{CONTROLLER MODEL OF FITNESS SIMU- LATION HORSE}

A PID controller based on the control theory was used to fully control the movement of fitness simulation horse, with the model shown as follows:

$u(\mathrm{k})=\mathrm{k}_{p}\left[\mathrm{e}(\mathrm{k})+\frac{T}{T_{i}} \sum_{j=1}^{k} e(\mathrm{j})+\mathrm{T}_{d} \frac{e(\mathrm{k})-e(\mathrm{k}-1)}{T}\right](26)$

Where, the differential time and integral time of the controller are $\mathrm{T}_{d}$ and $T_{i}$, the corresponding proportionality factor is $\mathrm{k}_{p}$, the deviation signal is $\mathrm{e}(\mathrm{t})$, the output is $u(\mathrm{k})$, and $T$ is the sample cycle.

In the equation above, suppose $\Delta e(\mathrm{k})=e(\mathrm{k})-e(\mathrm{k}-1)$ and simplify the calculation process, and then it can be drawn that:

$$
\begin{aligned}
& u(\mathrm{k})=\mathrm{k}_{p}\left[\mathrm{e}(\mathrm{k})+\frac{T}{T_{i}} \sum_{j=1}^{k} e(\mathrm{j})+\frac{\mathrm{T}_{d}}{T} \Delta e(\mathrm{k})\right](27) \\
& \text { From the above equation, if }
\end{aligned}
$$

$u(\mathrm{k})=\mathrm{k}_{p} \mathrm{e}(\mathrm{t})+\mathrm{k}_{i} T \sum_{j=1}^{k} e(\mathrm{j})+\mathrm{T}_{d} \frac{\mathrm{k}_{d}}{T} \Delta e(\mathrm{k})$

In order to avoid any deviation, the digital simulation controller model was actually used, as shown in the equation below:

$u(\mathrm{k}-1)=\mathrm{k}_{p}(\mathrm{e}(\mathrm{k}-1))$

$\left.+\mathrm{k}_{i} T \sum_{j=1}^{k} e(\mathrm{j})+k_{d}(e(\mathrm{k}-1)-\mathrm{e}(\mathrm{k}-2))\right) \frac{\mathrm{k}_{d}}{T} \Delta e(\mathrm{k})$

Then, suppose:

$\Delta u(\mathrm{k})=u(\mathrm{k})-u(\mathrm{k}-1)$
$\Delta e(\mathrm{k})=e(\mathrm{k})-e(\mathrm{k}-1)$

$\Delta e(\mathrm{k}-1)=e(\mathrm{k}-1)-e(\mathrm{k}-2)$

Thus, it can be drawn that from the subtraction between two equations above:

$$
\begin{aligned}
& \Delta u(\mathrm{k})=\mathrm{k}_{p}[\mathrm{e}(\mathrm{k})-e(\mathrm{k}-1)]+\mathrm{k}_{i} T e(\mathrm{k}) \\
& +\frac{\mathrm{k}_{d}}{T}[e(\mathrm{k})-2 e(\mathrm{k}-1)+e(\mathrm{k}-2)]
\end{aligned}
$$

As the fitness horse controller was improved, the error effect was avoided, so that the model precision was assured, the calculation became more efficient, and the storage space also decreased.

\section{EXPERIMENT OF FITNESS SIMULATION HORSE BASED ON CONTROL THEORY}

In order to validate the effectiveness of the controller for fitness simulation horse, a simulation experiment was conducted on this controller during its application in the fitness simulation horse. The comparison be- 


\section{MATEC Web of Conferences}

tween the traditional controller and digital simulation controller is shown in figure 4.

In addition, in order to observe whether the components in the model are proper linked, a dynamic simulation experiment was conducted on the fitness simulation horse, and the kinematic trajectories between the front and hind legs of fitness simulation horse and the contact points on the platform plane were drawn. As a man runs a circle, the fitness simulation horse also accordingly moves forward a cycle.

The kinematic trajectory for the front legs of fitness simulation horse is shown in Figure 5 given below:

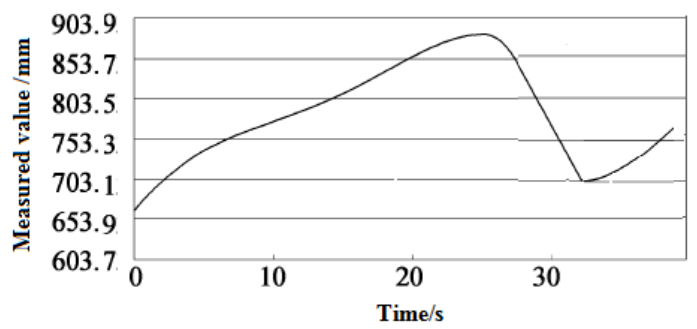

Figure 5. Displacement Curve of Front legs and Ground Contact Point

The kinematic trajectory for the hind legs of fitness simulation horse is shown in Figure 6 given below:

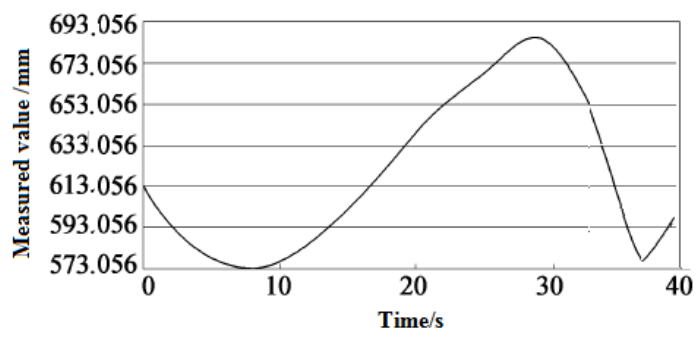

Figure 6. Displacement Curve of Hind Legs and Ground Contact Point

As seen from the simulation results above, the digital simulation controller is more stable and quickly responsive within less time than the traditional controller. During the movement trajectory test, the backward trajectory was quite level and smooth, and the postures of front and hind legs greatly varied. One of the break points occurring was because the tie rod was under great pressure when it rose to the highest point, and then a knee point appeared on the trajectory. The simulation results showed that, the fitness simulation horse can effectively run under the control from the controller based on the control theory.

\section{CONCLUSION}

In the environment of applying the parallel pipe robot structure based on new control theory, this paper designed the fitness simulation horse based on new control theory and effectively controlled the motion platform. The main work is listed below:

1) Propose the control theory and set up an orthogonal parallel platform for the fitness simulation horse by analyzing the advantages of fitness simulation horse to human health; effectively control the fitness simulation horse with the peripheral control system, so as to shorten the response time and improve the efficiency of the entire system.

2) Establish the kinematics model according to the platform characteristics. The final result was unique, which therefore assured the stability and improved the precision of the fitness simulation horse.

To sum up, this paper designed the fitness simulation horse based on the control theory and thus effectively controlled the system, which partly made progress in the control theory on fitness simulation horse. However, this control theory still remains to be studied and further completed before being applied into practice.

\section{REFERENCES}

[1] Zhao J.B. 2009. The Design of Freedom Degree Biomimetic Robot Horse System and the Study on Modeling Control. Yanshan University. 5: 9-15

[2] Zhang S.Q. 2014. Analysis of the Bionics Principle Application in Mechanical Design Domain $[\mathrm{C}] / / \mathrm{Si} \mathrm{Y}$. Science Times_-2014 Collected Papers of the Technical Innovation and Business Management Seminar Part I(Scientific and Technical Innovation). Beijing: Scientific Technology and Business: 52.

[3] Ren Y.Y. 2013. Research of the Physiological Information Fusion Algorithm and Its Application in Biomimetic Robot Horse. Yanshan University. 9: 5-10

[4] Zhang Z., Huang Q. \& Li G.R. 2008. The Analysis of 7DOF Humanoid Robot's Leg Motion and Its Simulation, Microcomputer Information. 2-2, 186-192.

[5] Wang H.R., Zhao J.B., Zhao L.X. \& Tian X.J. 2009. The Inverse-Kinematic Analysis of Biomimetic Robot Horse and Fuzzy PID Control. Microcomputer Information.

[6] S.Srikant. \& M.R. Akella. 2009. Persistence Filter-Based Control for Systems with Time-Varying Control Gains. Systems \& Control Letters, 58(6): 410-416

[7] Zadeh L A. 1972. A Rationale for Fuzzy Control. Trans. ASME J. Dynamic System Measure Control, 94: 5-7.

[8] Du P.D. 2013. Overview of Bionics and Biomechanics Study. Forestry Machinery \& Woodworking Equipment. 41(9): 15-19.

[9] Hwang. \& Yunn-Lin. 2006. Recursive Newton-Euler Formulation for Flexible Dynamic Manufacturing Analysis of Open-Loop Systems. International Journal of Advanced Manufacturing Technology, 29(5): 568-578. 